\title{
Impacto Atual da Circulação Extracorpórea na Cirurgia de Revascularização Miocárdica no Estado de São Paulo
}

\author{
Current Impact of Cardiopulmonary Bypass in Coronary Artery Bypass Grafting in São Paulo State
}

Gabrielle Barbosa Borgomoni, ${ }^{1 \oplus}$ Omar Asdrúbal Vilca Mejia, ${ }^{1,2}$ Bianca Maria Maglia Orlandi, ${ }^{1 \oplus}$ Maxim Goncharov, ${ }^{1 \oplus}$ Luiz Augusto Ferreira Lisboa, ${ }^{1}$ Pedro Henrique Conte, ${ }^{3 \oplus}$ Marco Antonio Praca Oliveira, ${ }^{3}$ Alfredo Inácio Fiorelli, ${ }^{1}$ Orlando Petrucci Junior, ${ }^{4}$ Marcos Grandim Tiveron, ${ }^{5}$ Luís Alberto de Oliveira Dallan, ${ }^{1}$ Fabio Biscegli Jatene ${ }^{1}$

Universidade de São Paulo - Faculdade de Medicina Hospital das Clínicas - Instituto do Coração, ${ }^{1}$ São Paulo, SP - Brasil

Hospital Samaritano Paulista, ${ }^{2}$ São Paulo, SP - Brasil

Beneficência Portuguesa de São Paulo, ${ }^{3}$ São Paulo, SP - Brasil

Universidade Estadual Campinas FCM Unicamp - Faculdade de Ciências Médicas, ${ }^{4}$ Campinas, SP - Brasil

Hospital Santa Casa de Misericórdia Marília, ${ }^{5}$ Marília, SP - Brasil

\section{Resumo}

Fundamento: Resultados prévios com o uso de circulação extracorpórea (CEC) geram dificuldades na escolha do melhor tratamento para cada paciente na cirurgia de revascularização miocárdica (CRM) no contexto atual.

Objetivo: Avaliar o impacto da CEC no cenário atual da CRM no estado de São Paulo.

Métodos: Foram analisados 2.905 pacientes submetidos à CRM de forma consecutiva em 11 centros do estado de São Paulo pertencentes ao Registro Paulista de Cirurgia Cardiovascular (REPLICCAR) I. Dados perioperatórios e de seguimento foram colocados via on-line por especialistas treinados e capacitados em cada hospital. Foram analisadas as associações das variáveis perioperatórias com o tipo de procedimento (com ou sem CEC) e com os desfechos. A mortalidade esperada foi calculada por meio do EuroSCORE II (ESII). Os valores de p menores de $5 \%$ foram considerados significativos.

Resultados: Não houve diferença significativa em relação à idade dos pacientes entre os grupos $(p=0,081)$. Dentre os pacientes, 72,9\% eram de sexo masculino; 542 pacientes foram operados sem CEC (18,7\%). Das características préoperatórias, pacientes com infarto agudo do miocárdio (IAM) prévio $(p=0,005)$ e disfunção ventricular $(p=0,031)$ foram operados com CEC; no entanto, pacientes de emergência ou em classe funcional New York Heart Association (NYHA) IV foram operados sem CEC $(p<0,001)$. O valor do ESII foi semelhante para ambos os grupos $(p=0,427)$. Na CRM sem CEC, houve preferência pelo uso do enxerto radial $(p<0,001)$ e com CEC pela artéria mamária direita $(p<0,001)$. No pós-operatório, $o$ uso de CEC esteve associado com reoperação por sangramento $(p=0,012)$.

Conclusão: Atualmente, no REPLICCAR, reoperação por sangramento foi o único desfecho associado ao uso da CEC na CRM. (Arq Bras Cardiol. 2020; 115(4):595-601)

Palavras-chave: Circulação Extracorpórea; Fatores de Risco; Revascularização Miocárdica; Epidemiologia; Mortalidade Hospitalar; Cuidados Pós-Operatórios; Morbidade.

\begin{abstract}
Background: Previous results on the use of cardiopulmonary bypass (CPB) have generated difficulties in choosing the best treatment for each patient undergoing myocardial revascularization surgery (CABG) in the current context.

Objective: Evaluate the current impact of CPB in CABG in São Paulo State.

Methods: A total of 2905 patients who underwent CABG were consecutively analyzed in 11 São Paulo State centers belonging to the São Paulo Registry of Cardiovascular Surgery (REPLICCAR) I. Perioperative and follow-up data were included online by trained specialists in each hospital. Associations of the perioperative variables with the type of procedure and with the outcomes were analyzed. The study outcomes were morbidity and operative mortality. The expected mortality was calculated using EuroSCORE II (ESII). The values of $p<5 \%$ were considered significant.
\end{abstract}

Omar Asdrúbal Vilca Mejia •

Universidade de São Paulo - Faculdade de Medicina Hospital das Clínicas - Instituto do Coração (INCOR). Avenida Dr. Enéas de Carvalho Aguiar, número 44, bloco II - 20 andar, sala 11 CEP- 05403-000 - São Paulo, SP - Brasil.

E-mail: omar.mejia@incor.com.usp.br

Artigo recebido em 13/03/2019, revisado em 12/08/2019, aceito em 18/08/2019

DOI: https://doi.org/10.36660/abc.20190145 
Results: There were no significant differences concerning the patients' age between the groups $(p=0.081) .72 .9 \%$ of the patients were males. Of the patients, 542 underwent surgery without CPB (18.7\%). Of the preoperative characteristics, patients with previous myocardial infarction $(p=0.005)$ and ventricular dysfunction $(p=0.031)$ underwent surgery with CPB. However, emergency or New York Heart Association (NYHA) class IV patients underwent surgery without CPB $(p<0.001)$. The ESII value was similar in both groups $(p=0.427)$. In $C A B G$ without $C P B$, the radial graft was preferred $(p<0.001)$, and in $C A B G$ with $C P B$ the right mammary artery was the preferred one $(p<0.001)$. In the postoperative period, CPB use was associated with reoperation for bleeding $(p=0.012)$.

Conclusion: Currently in the REPLICCAR, reoperation for bleeding was the only outcome associated with the use of CPB in CABG. (Arq Bras Cardiol. 2020; 115(4):595-601)

Keywords: Extracorporeal Circulation; Risk Factors; Myocardial Revascularization; lepidemiology; Hospital Mortality; Postoperative Care; Morbidity.

Full texts in English - http://www.arquivosonline.com.br

\section{Introdução}

A cirurgia de revascularização miocárdica (CRM) é um dos procedimentos mais estudados e, como consequência, foram alcançados excelentes resultados. ${ }^{1} \mathrm{O}$ advento da circulação extracorpórea (CEC), sem dúvidas, permitiu estabelecer a CRM como um tratamento seguro, efetivo e reproduzível; no entanto, sempre existiu uma preocupação quanto à influência da CEC na morbimortalidade. ${ }^{2}$ As primeiras análises que compararam CRM com e sem CEC foram em pacientes de baixo risco e não mostraram diferença significativa. ${ }^{3}$ Com o tempo, o controle da CEC passou a apresentar melhoras, e começaram a ser encontrados benefícios a curto prazo unicamente para subgrupos de maior risco. ${ }^{4}$ Entretanto, estudos randomizados mais atuais que não encontraram diferenças a curto prazo revelaram problemas com a técnica sem CEC, em que apontam complicações relacionadas com a patência das anastomoses e com os maiores índices de revascularização incompleta. ${ }^{5-7}$ Assim, embora existam critérios bem-definidos para indicação da CRM, a escolha da CEC continua sendo baseada no perfil clínico do paciente e na experiência do cirurgião. A oportunidade atual para a técnica sem CEC pode estar no aumento de pacientes frágeis encaminhados para CRM, ${ }^{8}$ no conceito de levar o procedimento correto para o paciente correto. Por outro lado, resultados atuais da CRM com CEC mostram uma diminuição da ocorrência de acidente vascular encefálico (AVE), ${ }^{9}$ embora problemas com o aumento de sangramento e disfunção renal ainda persistam em pacientes de alto risco.

No Brasil, a proporção de pacientes submetidos à CRM sem CEC é variável, assim como os resultados de morbimortalidade. ${ }^{10} \mathrm{~A}$ falta de uma diretriz nacional que oriente o manuseio da CEC por meio de ações dirigidas por metas, protocolos de segurança e sistemas de monitoramento em tempo real pode estar influenciando nos resultados; portanto, existe uma lacuna na nossa realidade sobre o impacto da CEC na CRM.

O objetivo deste estudo foi avaliar o impacto atual da prática da CEC na morbimortalidade dos pacientes submetidos à CRM do REPLICCAR, o maior registro de cirurgias cardiovasculares do estado de São Paulo.

\section{Métodos}

\section{Amostra}

A amostra total foi de 5.222 pacientes, dos quais 2.905 foram submetidos à CRM nas 11 instituições participantes do estudo REPLICCAR. ${ }^{11}$ Os pacientes foram operados consecutivamente, de novembro de 2013 a dezembro de 2016, nos seguintes hospitais:

1 - Instituto do Coração do Hospital das Clínicas da Faculdade de Medicina da Universidade de São Paulo (HC-FMUSP)

2 - Beneficência Portuguesa de São Paulo

3 - Hospital de Clínicas da Universidade Estadual de Campinas (Unicamp)

4 - Irmandade da Santa Casa de Piracicaba

5 - Irmandade da Santa Casa de São Paulo

6 - Hospital Paulo Sacramento de Jundiaí

7 - Hospital Pitangueiras do Grupo Sobam

8 - Hospital das Clínicas de Ribeirão Preto

9 - Hospital São Paulo da Universidade Federal de São Paulo (UNIFESP)

10 - Hospital de Base de São José do Rio Preto

11 - Irmandade da Santa Casa de Marília

\section{Critérios de Inclusão e Exclusão}

\section{Critérios de Inclusão}

Todos os pacientes com idade $\geq 18$ anos, operados de CRM de forma eletiva, urgência ou emergência no período estabelecido.

\section{Critérios de Exclusão}

Foram excluídos pacientes que realizaram, no mesmo procedimento, cirurgias associadas (incluindo valva, aorta torácica e outros) e procedimentos alternativos da insuficiência coronariana (laser, injeção de células-tronco e outros).

\section{Coleta, Definição e Organização dos Dados}

A análise foi realizada no banco de dados do REPLICCAR I. Trata-se de um registro prospectivo, multicêntrico e mandatório com coleta de dados entre novembro de 2013 e dezembro de 2016. A coleta de dados foi realizada por pessoas graduadas e treinadas para esta finalidade em cada centro participante. Os dados foram incorporados no site http://bdcardio.incor.usp.br, por meio de quatro interfaces disponíveis: pré-operatório, intraoperatório, pós-operatório e avaliação em 30 dias. O seguimento dos pacientes foi 
realizado por via telefônica. A completude e veracidade dos dados foram supervisionadas pelo comitê executivo do registro. As definições das variáveis foram adotadas a partir do EuroSCORE II, e o cálculo da mortalidade esperada no site http://www.EuroSCORE.org/calc.html.

Os desfechos analisados foram: morbidade (reoperação por sangramento, choque cardiogênico, AVE, infecção de sítio cirúrgico, mediastinite, pneumonia, infarto agudo do miocárdio [IAM] e insuficiência renal aguda [IRAg]) e mortalidade cirúrgica no período entre a cirurgia e a avaliação em 30 dias, ou, do contrário, até a alta hospitalar.

\section{Análise Estatística}

A análise estatística foi realizada com o uso do software STATA versão 13.1 (StataCorp, Texas, USA). Para avaliar a distribuição de normalidade dos dados, foi utilizado o Teste de Shapiro Wilk. As variáveis IMC ( $<18,5 \mathrm{~kg} / \mathrm{m} 2$ : baixo; 18,5 a 24,9kg/m2: normal; 25 a $29.9 \mathrm{~kg} / \mathrm{m} 2$ : sobrepeso e; $\geq 30 \mathrm{~kg} / \mathrm{m} 2$ : obesidade), classificação NYHA (I e II; III e IV), EuroSCORE II $(<5 \%$ e $\geq 5 \%)$, hemoglobina glicosilada $(\leq 7 \%$ e $>7 \%)$, fração de ejeção $(<30 \% ; 30$ a $50 \%$; $\geq 50 \%)$, hematócrito $(<30 \%$ e $\geq 30 \%)$ e creatinina $(\leq 1,4 \mathrm{mg} / \mathrm{dL}$ e $>1,4 \mathrm{mg} / \mathrm{dL})$ foram categorizadas como frequências absoluta e relativa, com o intervalo de confiança binomial de $95 \%$. Variáveis contínuas foram avaliadas quanto à diferença pelo teste de Mann Whitney, devido à distribuição dos dados. Contudo, para a comparação de categorias, utilizamos os testes qui-quadrado ou exato de Fisher. As variáveis contínuas assimétricas foram descritas através de mediana e intervalo interquartil. As variáveis de desfecho (complicações pós-operatórias) foram analisadas por meio da regressão logística univariada, e foram expressas a razão de chances (odds ratio) e o intervalo de confiança de $95 \%$. Os valores de $p$ menores de $5 \%$ foram considerados significativos.

\section{Ética e Termo de Consentimento}

Este trabalho foi uma subanálise dentro do projeto intitulado "Estratificação de Risco Cirúrgico como Instrumento de Inovação em Programas em Cirurgia Cardíaca no Sistema Único de Saúde do Estado de São Paulo" com registro on-line n 9696 da Comissão de Ética para Análises de Projetos de Pesquisa (CAPPesq) do HC-FMUSP.

\section{Resultados}

Foram avaliados 2.905 pacientes submetidos à CRM no período de estudo. Destes, $542(18,7 \%)$ não fizeram uso de CEC durante o procedimento. Não houve diferença significativa com relação à idade entre os grupos analisados $(p=0,081)$, a mediana dos pacientes com CEC foi de 63 anos (56 a 69), e sem CEC mediana, de 64 (56 a 71); 72,9\% foram do gênero masculino.

Na Tabela 1, estão descritas as características pré-operatórias dos grupos avaliados. Embora, os grupos demonstrem homogeneidade, observamos uma alta prevalência de infarto prévio do miocárdio em ambos os grupos (> de 40\%), sendo significativamente maior para o grupo de pacientes escolhidos para a cirurgia com CEC $(p=0,005)$. Não houve diferença significativa em relação ao valor da mediana do EuroSCORE II $(p=0,482)$ para ambos os grupos.

$\mathrm{Na}$ Tabela 2, podemos observar que pacientes com hemoglobina glicosilada $>7 \%$ foram operados com CEC $(p=0,008)$. Quando a limitação esteve relacionada com o coração, como são os pacientes com $\mathrm{FE}<50 \%$, eles foram escolhidos para cirurgia com CEC $(p=0,031)$. O tipo de intervenção não esteve associado com valores de hematócrito e creatinina para os vários pontos de corte analisados neste estudo.

No que diz respeito aos fatores intraoperatórios, na Tabela 3, verificamos que, quando escolhida a técnica sem CEC, em relação àquela com $\mathrm{CEC}$, houve maior utilização do enxerto de artéria radial $(p<0,001)$ do que enxerto de artéria torácica interna direita (ATID) $(p<0,001)$; no entanto, o uso de enxerto de artéria torácica interna esquerda (ATIE) não teve associação significativa $(p=0,276)$ com alguma das técnicas.

Com relação aos eventos pós-operatórios, na Tabela 4 , não identificamos associação significativa para a ocorrência de AVE em até 30 dias após a cirurgia, sendo a proporção semelhante entre os casos com e sem CEC $(p=0,473)$. O uso da CEC não esteve relacionado com a mortalidade cirúrgica $(p=0,761)$. No entanto, o uso da técnica com CEC esteve associado com reoperação por sangramento $(p=0,001)$, levando a um aumento do risco de sangramento de 6,2 vezes ( $\mathrm{B}=1,8$, IC95\% 0,41-3,23).

\section{Discussão}

Evidências mostram que a diminuição da resposta inflamatória na CRM sem CEC leva a uma redução de disfunção orgânica, ${ }^{2}$ assim como menores índices de vasoplegia e lesão renal. ${ }^{12}$ Neste cenário, análises retrospectivas em grandes populações confirmam uma redução significativa da morbimortalidade quando a CRM é realizada sem CEC..$^{13,14}$ Assim, mesmo uma análise nos quatro maiores centros dos EUA mostrou benefício quando a CRM foi realizada sem CEC, principalmente nos pacientes considerados de alto risco. ${ }^{15}$ Dois estudos publicados no mesmo período, um nos EUA $^{16}$ e outro no Brasil, ${ }^{17}$ revelaram também um maior risco de óbito nos pacientes operados com CEC em comparação aos operados sem CEC, principalmente no grupo de alto risco. Da mesma forma, uma análise de 30 anos da CRM sem CEC demonstrou uma redução significativa nos desfechos de mortalidade hospitalar, acidente cerebrovascular, complicações pós-operatórias graves, tempo de hospitalização e diminuição de custos. ${ }^{18}$

No entanto, estudos randomizados de grande impacto não mostraram diferença a favor da CRM sem CEC no que diz respeito à morbimortalidade. ${ }^{19-21}$ Em nossa análise, com uma amostra atual e multicêntrica, a única diferença encontrada a favor da CRM sem CEC foi o menor número de reoperações por sangramento. Isso também foi verificado no estudo de Lamy et al.,22 onde mesmo não existindo uma diferença significativa na morbimortalidade, houve uma diminuição na taxa de reoperação por sangramento. A taxa de reoperação por sangramento encontrada em nosso estudo é similar à encontrada em outro estudo, ${ }^{23}$ mas, em contrapartida, a mortalidade foi 4,5 vezes maior nos pacientes que tiveram essa complicação. 


\section{Artigo Original}

Tabela 1 - Características pré-operatórias da amostra de pacientes submetidos à CRM com e sem CEC. REPLICCAR, São Paulo, 2019

\begin{tabular}{|c|c|c|c|c|c|c|}
\hline \multicolumn{7}{|c|}{ CEC } \\
\hline \multirow[t]{2}{*}{ Características } & \multicolumn{2}{|c|}{$\underset{\substack{\operatorname{Sim} \\
(\mathrm{n}=2.363)}}{ }$} & \multicolumn{2}{|c|}{$\begin{array}{c}\text { Não } \\
(n=542)\end{array}$} & \multirow[t]{2}{*}{ IC $95 \%$} & \multirow{2}{*}{ Valor de $p$} \\
\hline & $\mathrm{N}$ & $\%$ & $\mathrm{~N}$ & $\%$ & & \\
\hline Idade, mediana (IIQ) & \multicolumn{2}{|c|}{$63(56-69)^{*}$} & \multicolumn{2}{|c|}{$64(56-71)^{*}$} & 62,4 a 62,6 & $0,081 \dagger$ \\
\hline Gênero & & & & & 0,25 a 0,29 & $0,125 \ddagger$ \\
\hline Masculino & 1.737 & 73,5 & 381 & 70,3 & & \\
\hline Feminino & 625 & 26,5 & 161 & 29,7 & & \\
\hline Índice de massa corporal & & & & & 27,3 a 27,6 & $0,809 \ddagger$ \\
\hline$<18,5$ & 14 & 0,6 & 4 & 0,7 & & \\
\hline 18,5 a 24,9 & 709 & 30 & 173 & 31,9 & & \\
\hline 25 a 29,9 & 1.057 & 44,7 & 234 & 43,2 & & \\
\hline$\geq 30$ & 583 & 24,7 & 131 & 24,2 & & \\
\hline $\begin{array}{l}\text { Infarto prévio do } \\
\text { miocárdio }\end{array}$ & 1.142 & 48,3 & 226 & 41,7 & 0,45 a 0,49 & $0,005 \ddagger$ \\
\hline Stent prévio & 389 & 16,5 & 98 & 18,1 & 0,15 a 0,18 & $0,363 \ddagger$ \\
\hline Cirurgia cardíaca prévia & 36 & 1,5 & 4 & 0,7 & 0,01 a 0,02 & $0,157 \ddagger$ \\
\hline Insulinodependente & 382 & 16,2 & 96 & 17,7 & 0,15 a 0,18 & $0,381 \ddagger$ \\
\hline Doença pulmonar obstrutiva crônica & 17 & 0,7 & 4 & 0,7 & 0,004 a 0,01 & $0,963 \ddagger$ \\
\hline Angina classe funcional IV & 442 & 18,7 & 91 & 16,8 & 0,17 a 0,20 & $0,299 \ddagger$ \\
\hline NYHA & & & & & 0,32 a 0,36 & $0,917 \ddagger$ \\
\hline | e || & 1.562 & 66,1 & 357 & 65,9 & & \\
\hline III e IV & 801 & 33,9 & 185 & 34,1 & & \\
\hline EuroSCORE II & & & & & 0,07 a 0,09 & $0,482 \ddagger$ \\
\hline$<5 \%$ & 2.156 & 91,5 & 501 & 92,4 & & \\
\hline$\geq 5 \%$ & 200 & 8,5 & 41 & 7,6 & & \\
\hline
\end{tabular}

*Mediana e intervalo interquartil (IIQ); † Mann Whitney; $¥$ Teste qui-quadrado ou exato de Fisher.

A prevalência de CRM sem CEC na nossa amostra é similar aos valores relatados em outras casuísticas, ${ }^{24}$ sugerindo a aderência às diretrizes na escolha da técnica e a inclusão de todos os pacientes no registro. Na análise, também foi evidenciado que, quando as limitações cirúrgicas estiveram relacionadas à manipulação do coração, como infarto prévio e/ou disfunção ventricular, a equipe tendeu a escolher a técnica com CEC. No entanto, bons resultados também foram encontrados quando operados sem CEC. ${ }^{25}$ Por outro lado, quando as limitações estiveram relacionadas com a gravidade do paciente, tipo NYHA IV ou estado de emergência, a escolha foi pela técnica sem CEC. Isso corrobora com estudos que mostram uma preferência pela CRM sem CEC nos pacientes instáveis. ${ }^{26} \mathrm{~A}$ maior utilização da artéria radial na CRM sem CEC pode ser explicada pelo menor tempo para obtenção do enxerto em relação à dupla mamária principalmente nos quadros agudos.

Cantero et al., ${ }^{27}$ publicaram uma mortalidade hospitalar de $4,3 \%$ e $4,7 \%$, respectivamente, no grupo sem CEC e com CEC $(p=0,92)$, similar aos valores encontrados no nosso estudo $(p=0,76)$. No entanto, diferentemente do nosso estudo, eles encontraram nos pacientes operados sem CEC um menor índice de complicações em relação ao infarto $(p=0,02)$ e ao uso de balão intra-aórtico $(p=0,01)$.

Neste estudo, não encontramos correlação significativa no que diz respeito ao gênero feminino com maiores índices de desfechos negativos. Como descrito por Sá et al., ${ }^{28}$ isso talvez esteja relacionado com o tamanho amostral dos diferentes estudos.

Os escores de risco são instrumentos de predição que ajudam pacientes e profissionais da saúde na tomada de decisões sobre o provável risco de complicações ou óbito. Em um estudo realizado no InCor-HC-FMUSP, foi encontrado um ponto de corte para o EuroSCORE e o 2000BP que ajudaria na tomada de decisões para o uso ou não da CEC na CRM. ${ }^{17}$ Em nosso estudo, foi utilizado o EuroSCORE II, o mesmo que subestimou a nossa mortalidade observada, o que contraindicaria sua utilização para a tomada de decisões na amostra estudada. Isso confirma as orientações da última diretriz europeia, em que o uso atual do ESII para predição de mortalidade após CRM fica contraindicado. ${ }^{29}$ 
Artigo Original

Tabela 2 - Avaliação pré-operatória da amostra de pacientes submetidos à CRM com e sem CEC. REPLICCAR, São Paulo, 2019

\begin{tabular}{|c|c|c|c|c|c|c|}
\hline \multirow{3}{*}{ Exames pré } & \multicolumn{4}{|c|}{ CEC } & \multirow[b]{3}{*}{ IC $95 \%$} & \multirow{3}{*}{ Valor de $p$} \\
\hline & \multicolumn{2}{|c|}{$\operatorname{Sim}(n=2363)$} & \multicolumn{2}{|c|}{ Não $(n=542)$} & & \\
\hline & $\mathrm{n}$ & $\%$ & $\mathrm{~N}$ & $\%$ & & \\
\hline Hemoglobina glicosilada & & & & & 6,6 a 6,9 & $0,008 \ddagger$ \\
\hline$\leq 7 \%$ & 784 & 68,0 & 159 & 77,0 & & \\
\hline$>7 \%$ & 369 & 32,0 & 47 & 23,0 & & \\
\hline Fração de ejeção & & & & & 56,5 a 57,3 & $0,031 \ddagger$ \\
\hline$<30 \%$ & 36 & 1,5 & 4 & 0,7 & & \\
\hline 30 a $50 \%$ & 474 & 20,1 & 87 & 16,1 & & \\
\hline$\geq 50 \%$ & 1853 & 78,4 & 451 & 83,2 & & \\
\hline Hematócrito & & & & & 39,9 a 40,3 & $0,218 \ddagger$ \\
\hline$\geq 30 \%$ & 2284 & 96,7 & 518 & 95,6 & & \\
\hline$<30 \%$ & 79 & 3,3 & 24 & 4,4 & & \\
\hline Creatinina & & & & & 1,1 a 1,2 & $0,651 \ddagger$ \\
\hline$\leq 1,4 \mathrm{mg} / \mathrm{dL}$ & 2049 & 86,7 & 466 & 86,0 & & \\
\hline$>1,4 \mathrm{mg} / \mathrm{dL}$ & 314 & 13,3 & 76 & 14,0 & & \\
\hline
\end{tabular}

Tabela 3 - Fatores intraoperatórios da amostra de pacientes submetidos à CRM com e sem CEC. REPLICCAR, São Paulo, 2019

\begin{tabular}{|c|c|c|c|c|c|c|}
\hline \multirow{3}{*}{ Intraoperatório } & \multicolumn{4}{|c|}{ CEC } & \multirow{3}{*}{ IC $95 \%$} & \multirow{3}{*}{ Valor de } \\
\hline & \multicolumn{2}{|c|}{$\operatorname{Sim}(n=2363)$} & \multicolumn{2}{|c|}{ Não $(n=542)$} & & \\
\hline & $\mathrm{n}$ & $\%$ & $\mathrm{n}$ & $\%$ & & \\
\hline ATIE & 2.221 & 94 & 516 & 95,2 & 0,93 a 0,95 & $0,276 \ddagger$ \\
\hline ATID & 282 & 11,9 & 30 & 5,5 & 0,09 a 0,12 & $<0,001 \ddagger$ \\
\hline Radial & 134 & 5,7 & 114 & 21 & 0,08 a 0,1 & $<0,001 \ddagger$ \\
\hline
\end{tabular}

$\ddagger$ Teste qui-quadrado ou exato de Fisher.

Tabela 4 - Regressão logística univariada de complicações pós-operatórias da amostra de pacientes submetidos a cirurgia de Revascularização do Miocárdio com e sem CEC. REPLICCAR, São Paulo, 2019

\begin{tabular}{lccccc}
\hline Desfechos clínicos e mortalidade & \multicolumn{2}{c}{ Uso de CEC } & & \\
\hline & Sim $(\mathbf{n}=\mathbf{2 3 6 3})$ & Não $(\mathbf{n}=\mathbf{5 4 2})$ & OR & IC95\% & Valor de $\mathbf{p}$ \\
\hline Reoperação por sangramento & $53(2,2)$ & $2(0,4)$ & 6,2 & 1,5 a 25,5 & 0,012 \\
Choque cardiogênico & $77(3,3)$ & $20(3,7)$ & 0,88 & 0,53 a 1,45 & 0,614 \\
AVE & $19(0,8)$ & $5(0,9)$ & 0,87 & 0,32 a 2,3 & 0,784 \\
Infecção do sítio cirúrgico & $286(12,1)$ & $55(10,2)$ & 1,2 & 0,9 a 1,7 & 0,203 \\
Mediastinite & $16(0,7)$ & $6(1,1)$ & 0,61 & 0,24 a 1,6 & 0,303 \\
Pneumonia & $163(6,9)$ & $30(5,5)$ & 1,3 & 0,85 a 1,89 & 0,251 \\
IAM & $38(1,6)$ & $12(2,2)$ & 0,72 & 0,37 a 1,4 & 0,330 \\
IRAg & $118(5,0)$ & $32(5,9)$ & 0,84 & 0,56 a 1,25 & 0,338 \\
Óbito & $102(4,3)$ & $25(4,6)$ & 0,93 & 0,57 a 1,46 & 0,761 \\
\hline
\end{tabular}

OR: Odds ratio; AVE: acidente vascular encefálico; IAM: infarto agudo do miocárdio; IRAg: insuficiência renal aguda. 
As limitações deste estudo foram: (1) a influência das variações no manuseio da CEC e dos protocolos sem CEC utilizados em cada centro participante não foi analisada. (2) Não foram analisados aspectos importantes, como o uso de antiagregantes plaquetários e o uso de antifibrinolíticos nos pacientes submetidos à CRM. No entanto, sabemos que o uso de protocolos seguindo as evidências atuais têm reduzido consideravelmente o aumento do risco de sangramento. ${ }^{30,31}$

Em resumo, ensaios clínicos randomizados não encontraram, em curto prazo, reduções na morbidade e mortalidade demonstradas nos estudos observacionais quando a CRM foi realizada sem CEC. No futuro, o uso de uma CEC mais monitorada e em tempo real, incluindo gasometria online e terapia dirigida por metas, pode ressaltar as vantagens do uso da CEC. Finalmente, é importante reiterar que o estado da arte atual é que grupos multidisciplinares definam e escolham a técnica correta para o paciente certo.

\section{Conclusão}

Pacientes escolhidos para CRM com CEC, foram os mais estáveis clinicamente, porém, os com pior função ventricular em comparação aos operados sem CEC. A reoperação por sangramento foi o único desfecho associado à prática atual da CEC na CRM no estado de São Paulo; contudo, tal complicação não influenciou no aumento do número de óbitos.

\section{Referências}

1. LaPar DJ, Filardo G, Crosby IK, Speir AM, Rich JB, Kron IL, et al. The challenge of achieving $1 \%$ operative mortality for coronary artery bypass grafting: a multi-institution Society of Thoracic Surgeons Database analysis. J Thorac Cardiovasc Surg. 2014;148(6):2686-96.

2. Weiland AP, Walker WE. Physiologic principles and clinical sequelae of cardiopulmonary bypass. Heart Lung. 1986;15(1):34-9.

3. Buffolo E, Andrade JCS, Succi JE, Leão LEV, Branco JNR, Cueva C, et al. Direct myocardial revascularization without extra-corporeal circulation: results in 391 patients. Rev Bras Cir Cardiovasc. 1986;1(1):32-9.

4. Puskas JD, Edwards FH, Pappas PA, O'Brien S, Peterson ED, Kilgo P, et al. Off-pump techniques benefit men and women and narrow the disparity in mortality after coronary bypass grafting. Ann Thorac Surg. 2007;84(5):1447-56.

5. Angelini GD, Culliford L, Smith DK, Hamilton MC, Murphy GJ, Ascione R, et al. Effects of on- and off-pump coronary artery surgery on graft patency, survival and quality of life: long term follow-up of two randomised controlled trials. J Thorac Cardiovasc Surg. 2009;137(2):295-303.

6. Legare JF, Buth KJ, King S, Wood J, Sullivan JA, Hancock Friesen C, et al. Coronary bypass surgery performed off pump does not result in lower inhospital morbidity than coronary artery bypass grafting performed on pump. Circulation. 2004;109(7):887-92.

7. Shroyer AL, Grover FL, Hattler B, Collins JF, McDonald GO, Kozora E, et al. On-pump versus off-pump coronary-artery bypass surgery. N Engl J Med. 2009 Nov 5;361:1827-37.

8. Mejía OAV, Sá MPBO, Deininger MO, Dallan LRP, Segalote RC, Oliveira MAP, et al. Off-pump versus On-pump Coronary Artery Bypass Grafting in Frail Patients: Study Protocol for the FRAGILE Multicenter Randomized Controlled Trial. Braz J Cardiovasc Surg. 2017;32(5):428-34.

\section{Contribuição dos Autores}

Concepção e desenho da pesquisa: Borgomoni GB, Mejia OAV, Lisboa LAF, Conte PH, Oliveira MAP, Petrucci Junior O, Tiveron M; Obtenção de dados: Borgomoni GB, Mejia OAV, Conte PH, Oliveira MAP, Petrucci Junior O, Tiveron M, Dallan LAO, Jatene FB; Análise e interpretação dos dados: Borgomoni GB, Mejia OAV, Goncharov M, Lisboa LAF, Conte PH, Oliveira MAP, Fiorelli AI, Petrucci Junior O, Tiveron M, Dallan LAO; Análise estatística: Borgomoni GB, Mejia OAV, Orlandi BMM, Goncharov M; Redação do manuscrito: Borgomoni GB; Revisão crítica do manuscrito quanto ao conteúdo intelectual importante: Mejia OAV, Lisboa LAF, Dallan LAO, Jatene FB.

\section{Potencial Conflito de Interesses}

Declaro não haver conflito de interesses pertinentes.

\section{Fontes de Financiamento}

O presente estudo foi financiado pelo FAPESP.

\section{Vinculação Acadêmica}

Não há vinculação deste estudo a programas de pósgraduação.
9. Tarakji KG, Sabik JF 3rd, Bhudia SK, et al. Temporal onset, risk factors, and outcomes associated with stroke after coronary artery bypass grafting. JAMA. 2011;305(4):381-90.

10. Piegas, L S, Bittar OJN, HaddadN.Myocardial Revascularization Surgery (MRS). Results from National Health System (SUS). Arq Bras Cardiol. 2009;93(5):555-60.

11. Mejía OAV, Lisboa LAF, Dallan LAO, Pomerantzeff PMA, Trindade EM, Jatene $\mathrm{FB}$, et al. Heart surgery programs innovation using surgical risk stratification at the São Paulo State Public Healthcare System: SP-SCORE-SUS STUDY. Rev Bras Cir Cardiovasc. 2013;28(2):263-9.

12. Hossne Junior NA, Miranda M, Monteiro MR, Branco JNR, Vargas GF, Pestana JOMA, et al. Cardiopulmonary bypass increases the risk of vasoplegic syndrome after coronary artery bypass grafting in patients with dialysisdependent chronic renal failure. Rev Bras Cir Cardiovasc. 2015;30(4):482-8.

13. Hannan EL, Wu C, Smith CR, Higgins RS, Carlson RE, Culliford AT, et al. Offpump versus on-pump coronary artery bypass graft surgery: differences in short-term outcomes and in long-term mortality and need for subsequent revascularization. Circulation. 2007;116(10):1145-52.

14. Puskas JD, Edwards FH, Pappas PA, O'Brien S, Peterson ED, Kilgo P, et al. Offpump techniques benefit men and women and narrow the disparity in mortality after coronary bypass grafting. Ann Thorac Surg. 2007;84(5):1447-56.

15. Mack MJ, Pfister A, Bachand D, Emery R, Magee MJ, Connolly M, et al. Comparison of coronary bypass surgery with and without cardiopulmonary bypass in patients with multivessel disease. J Thorac Cardiovasc Surg. 2004;127(1):167-73.

16. Polomsky M, He X, O’Brien SM, Puskas JD. Outcomes of off-pump versus on-pump coronary artery bypass grafting: impact of preoperative risk. J Thorac Cardiovasc Surg. 2013;145(5):1193-8. 
17. Mejıa OA, Lisboa LA, Puig LB, Moreira LF, Dallan LA, Jatene FB. On-pump or off-pump? Impact of risk scores in coronary artery bypass surgery. Rev Bras Cir Cardiovasc. 2012;27(4):503-11.

18. Buffolo E, Lima RC, Salerno TA. Myocardial revascularization without cardiopulmonary bypass: historical background and thirty-year experience. Rev Bras Cir Cardiovasc. 2011;26(3):3-7.

19. Moller CH, Penninga L, Wetterslev J, Steinbruchel DA, Gluud C. Clinical outcomes in randomized trials of off- vs. on-pump coronary artery bypass surgery: systematic review with meta-analyses and trial sequential analyses. Eur Heart J. 2008;29(21):2601-16.

20. Shroyer AL, Grover FL, Hattler B, Collins JF, McDonald GO, Kozora E, et al.. On-pump versus off-pump coronary-artery bypass surgery. N Engl J Med. 2009;361(19):1827-37.

21. Diegeler A, Borgermann J, Kappert V, Breuer M, Boning A, Ursulescu A, et al. Off-pump versus on-pump coronary-artery bypass grafting in elderly patients. N Engl J Med. 2013;368(13):1189-98.

22. Lamy A, Devereaux PJ, Prabhakaran D, Taggart DP, Hu S, Paolasso E, et al. Off-pump or on-pump coronary-artery bypass grafting at 30 days. N Engl J Med. 2012;366(16):1489-97.

23. Mehta RH, Sheng S, O'Brien SM, Grover FL, Gammie JS, Ferguson TB, et al. Reoperation for bleeding in patients undergoing coronary artery bypass surgery: incidence, risk factors, time trends, and outcomes. Circ Cardiovasc Qual Outcomes. 2009;2(6):583-90.

24. Bakaeen FG, Shroyer AL, Gammie JS, Sabik JF, Cornwell LD, Coselli JS, et al. Trends in use of off-pump coronary artery bypass grafting: results from the Society of Thoracic Surgeons Adult Cardiac Surgery Database. J Thorac Cardiovasc Surg. 2014;148(3):856-3

25. Caputti GM, Palma JH, Gaia DF, Buffolo E. Off-pump coronary artery bypass surgery in selected patients is superior to the conventional approach for patients with severely depressed left ventricular function. Clinics. 2011;66(12):2049-53.

26. Fattouch K, Guccione F, Dioguardi P, Sampognaro R, Corrado E, Caruso M, et al: Off-pump versus on-pump myocardial revascularization in patients with ST-segment elevation myocardial infarction: A randomized trial. J Thorac Cardiovasc Surg. 137(3):650-6.27.

27. Cantero MA, Almeida RM, Galhardo R. Analysis of immediate results of on-pump versus off-pump coronary artery bypass grafting surgery. Rev Bras Cir Cardiovasc. 2012;27(1):38-44.

28. Sá MPBO, Lima LP, Rueda FG, Escobar RR, Cavalcanti PEF, Thé ECS, et al Estudo comparativo entre cirurgia de revascularização miocárdica com e sem circulação extracorpórea em mulheres. Rev Bras Cir Cardiovasc. 2010;25(2):238-44

29. Neumann FJ, Sousa-Uva M, Ahlsson A, Alfonso F, Banning AP, Benedetto $\mathrm{U}$, et al. 2018 ESC/EACTS guidelines on myocardial revascularization. Eur Heart J. 2018;40(2):87-165

30. Berger JS, Frye CB, Harshaw Q, Edwards FH, Steinhubl SR, Becker RC. Impact of clopidogrel in patients with acute coronary syndromes requiring coronary artery bypass surgery: a multicenter analysis. J Am Coll Cardiol. 2008;52(21):1693-701.

31. Myles PS, Smith JA, Forbes A, Silbert B, Jayarajah M, Painter T, et al. Tranexamic acid in patients undergoing coronary-artery surgery. N Engl J Med. 2017;376(2):136-48. 